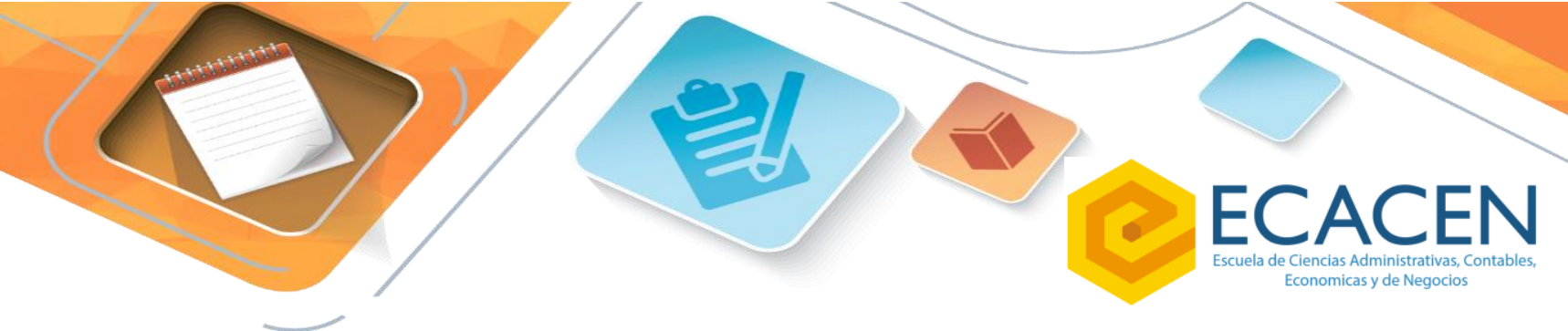

\title{
DIAGNÓSTICO DEL PERFIL EMPRENDEDOR DE LOS ESTUDIANTES DE LAS I.E.M. DEL SECTOR RURAL DE PASTO
}

\section{NOMBRE DE AUTORES}

\author{
Carlos Rubén Trejos Moncayo ${ }^{1}$
}

German Alfredo López Montezuma²

Luz Elena Montoya Rendón ${ }^{3}$

\section{RESUMEN}

En el marco del proyecto titulado: Diseño instruccional como alternativa para el fomento del emprendimiento en las I.E.M del sector rural de Pasto, adelantado por el grupo de investigación Sindamanoy de la ECACEN, se desarrolló un acercamiento a seis (6) instituciones educativas para conocer el perfil de los estudiantes, a partir del cual se pueda evidenciar las tendencias principales de la cultura del emprendimiento y finalmente, diagnosticar el estado actual de las actitudes emprendedoras, desde la Guía no. 39 del M.E.N.

Los resultados y su correspondiente interpretación se presentan en el siguiente documento, donde se asumieron como variables principales la jornada de estudio,

\footnotetext{
${ }^{1}$ Administrador de Empresas, Especialista en Gerencia Estratégica de Mercadeo, Magister en Mercadeo Agroindustrial, Docente de la Escuela de Ciencias Administrativas Contables Económicas y de Negocios ECACEN de la UNAD, investigador grupo Sindamanoy, líneas de investigación: emprendimiento y cadenas productivas. Correo electrónico carlos.trejos@unad.edu.co, C.C. No. 98394180 de Pasto. Código ORCID http://orcid.org/0000-0003-0352-9981

2 Administrador Financiero, Especialista en Finanzas, Magister en Docencia Universitaria, Docentes Asistente de la Escuela de Ciencias Administrativas Contables Económicas y de Negocios ECACEN de la UNAD, investigador grupo Sindamanoy, líneas de investigación emprendimiento, prospectiva y cadenas productivas. Correo electrónico german.lopez@unad.edu.co, C.C.12978110 de Pasto, Cel. 3012864934. Código ORCID http://orcid.org/0000-0002-0399-6363

${ }^{3}$ Administradora de Empresas, Maestría en Administración de Empresas con Énfasis en Sistemas Integrados de Gestión. Investigador asociado del Grupo de Investigación Ilama. Docente de la Universidad Nacional Abierta y a Distancia - UNAD, Colombia. luz.montoya@unad.edu.co
} 


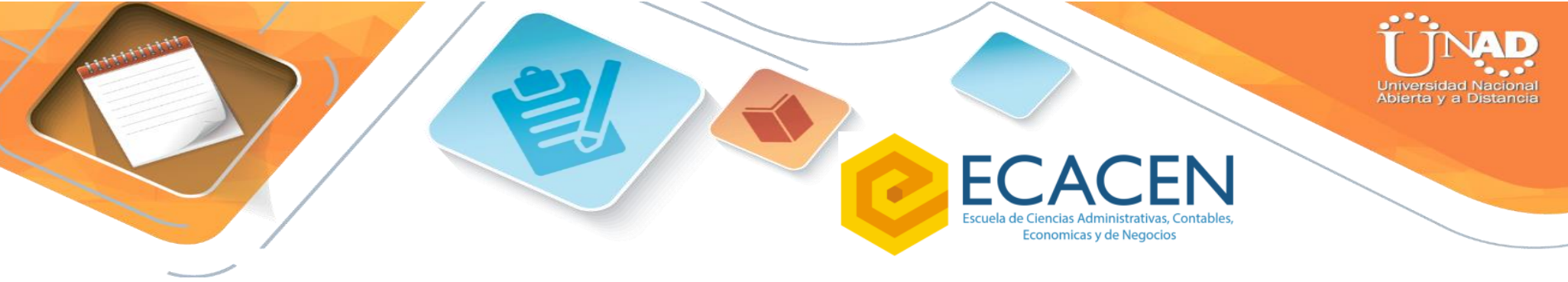

or unfavorableness of the entrepreneurial attitudes of the students of the I.E.M of the rural sector of Pasto.

Keywords: Municipal educational institutions; culture; entrepreneurship; student.

\section{INTRODUCCIÓN}

Al buscar la definición sobre emprendimiento, la Guía 39 del Ministerio de Educación Nacional (2012), menciona que "el emprendimiento, desde un enfoque de desarrollo humano integral es entendido como una forma de pensar, sentir y actuar para la creación de valor, lo cual permite a la comunidad educativa proponer espacios y escenarios de formación". Esto es evidente, porque se identifica la necesidad de construir conocimientos, fortalecer hábitos, valores, creencias y actitudes, muy necesarios para generar acciones en el fortalecimiento de capacidades y el desarrollo humano integral en temas de emprendimiento.

Este documento presenta un acercamiento a la caracterización del perfil de los estudiantes del grado onceavo, de los seis principales establecimientos educativos de las veredas y corregimientos del municipio de Pasto. Por lo anterior, se contempla como objetivo central, conocer los avances en formación de la cultura del emprendimiento en las IEM del sector rural de Pasto, donde inicialmente se realizó un diagnóstico de los aspectos que influyen directa o indirectamente con la cultura del emprendimiento como proceso de formación en 142 estudiantes matriculados en las Instituciones objeto de estudio.

Por lo anterior, es necesario describir los aspectos que están estrechamente relacionados con la jornada de trabajo de los estudiantes, el género, su edad promedio, el estrato socioeconómico del núcleo familiar, la situación laboral actual de los estudiantes encuestados, el máximo nivel de estudios del padre y madre, el grupo étnico al que pertenece, entre otros. Información, que permite conocer las capacidades, desde diferentes dimensiones del ser humano, que coadyuvan a generar acciones en todos los ámbitos o etapas de los estudiantes, en pro de su bienestar y progreso. 


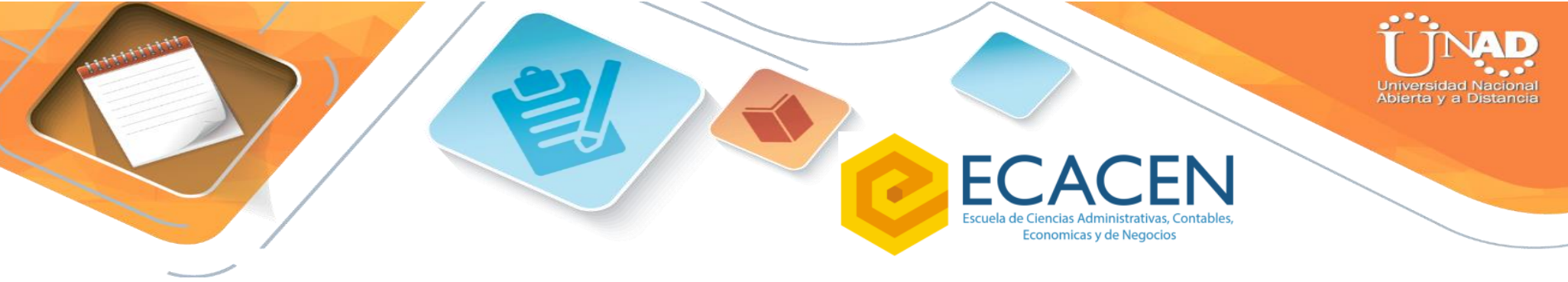

Otro de los criterios que caracterizan al grupo de estudiantes participantes en la investigación, hace referencia al género y promedio de edad como un elemento que para nuestro proceso permite conocer a la población estudiantil del sector rural de Pasto. De los 142 estudiantes indagados, un número de 75 estudiantes correspondiente al $53 \%$ pertenecen al género femenino, los 67 restantes corresponden al género masculino. Ahora bien, en lo referente a la edad los estudiantes que aceptan participar en la investigación se encuentran en un rango entre 15 y 20 años, el porcentaje predominante tiene una edad de 19 años representando el $27 \%$ del total de la muestra.

Al respecto, Howard Gardner (1998), manifiesta que se hace necesario tener en cuenta todas las inteligencias humanas dentro y fuera de las aulas. Puesto que todos somos diferentes, en gran parte porque todos tenemos distintas combinaciones de inteligencias, las cuales requieren diseñar métodos de evaluación que tengan en cuenta las diferencias.

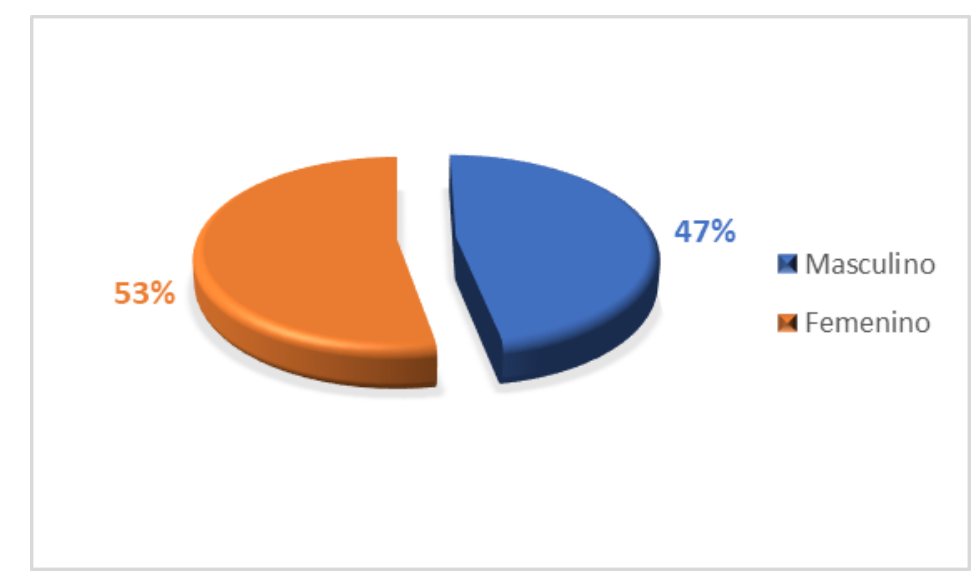

Gráfica 2. Género de los estudiantes 


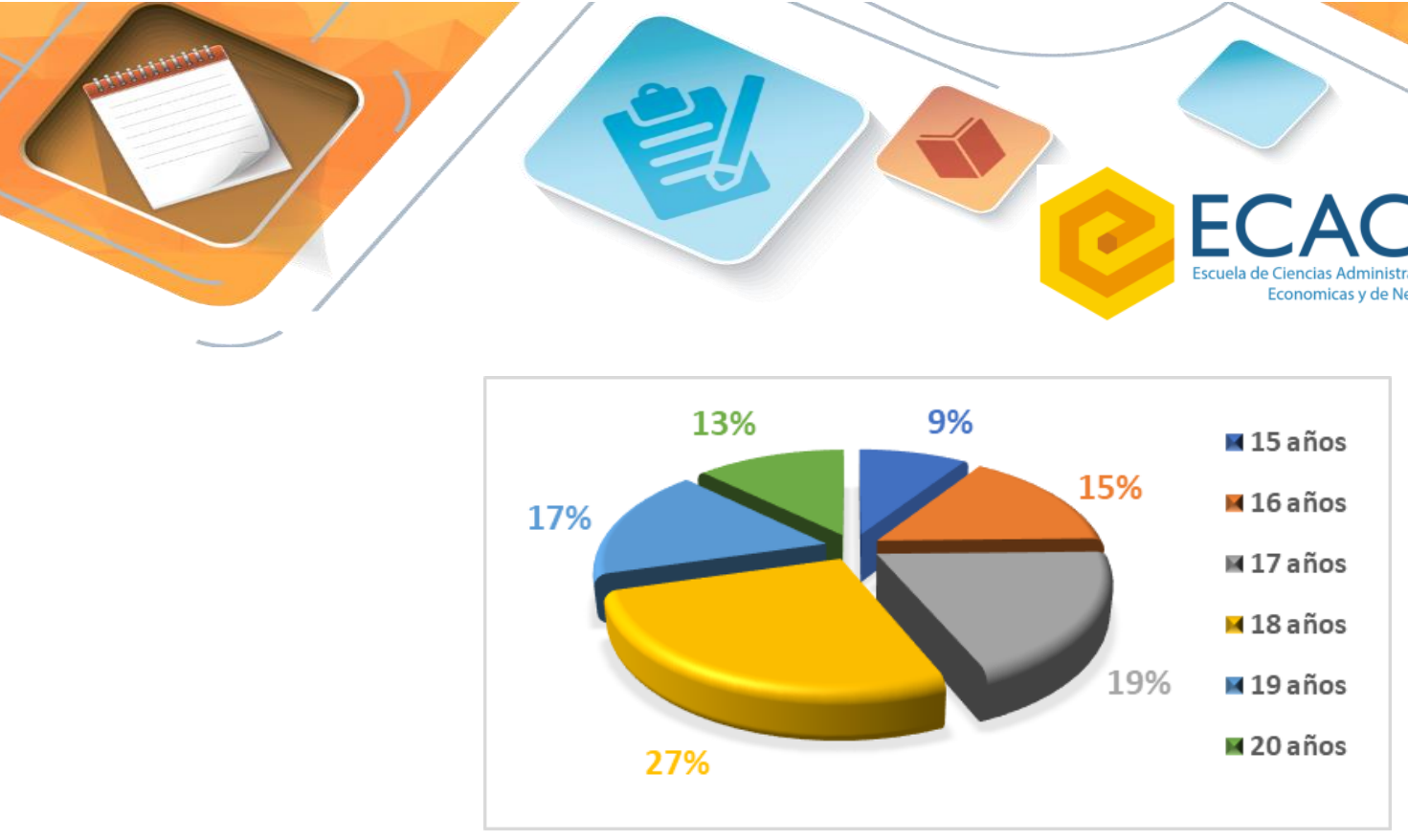

\section{Gráfica 3. Edad promedio}

Siendo la intención caracterizar a los estudiantes que participan del proceso de formación educativa y de manera específica conocer los avances en el desarrollo de la cultura del emprendimiento al interior de las 6 Instituciones Educativas Municipales del sector rural, se procede a consultar el estrato económico del lugar donde reside cuyo resultado muestra que el $61 \%$ de los 142 estudiantes residen en una vivienda de estrato 1 , el $29 \%$ residen en una vivienda de estrato 2 , el número restante del grupo indagado manifiesta que la residencia se cataloga con un estrato 0.

Para Guerrero y Delgado (2015), esta población afronta dos grandes dificultades a la hora de conseguir su primer trabajo. Por una parte, el bajo nivel de estudios y por otro lado la falta de experiencia, principalmente en aquellas ocupaciones que demandan competencias funcionales o destrezas técnicas.

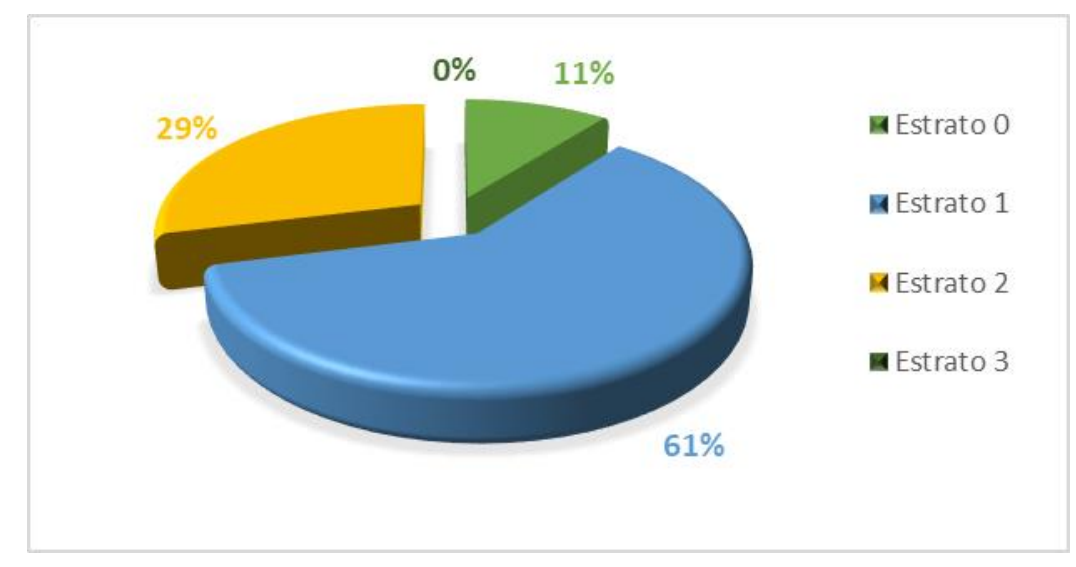

Gráfica 4. Estrato socioeconómico del núcleo familiar 


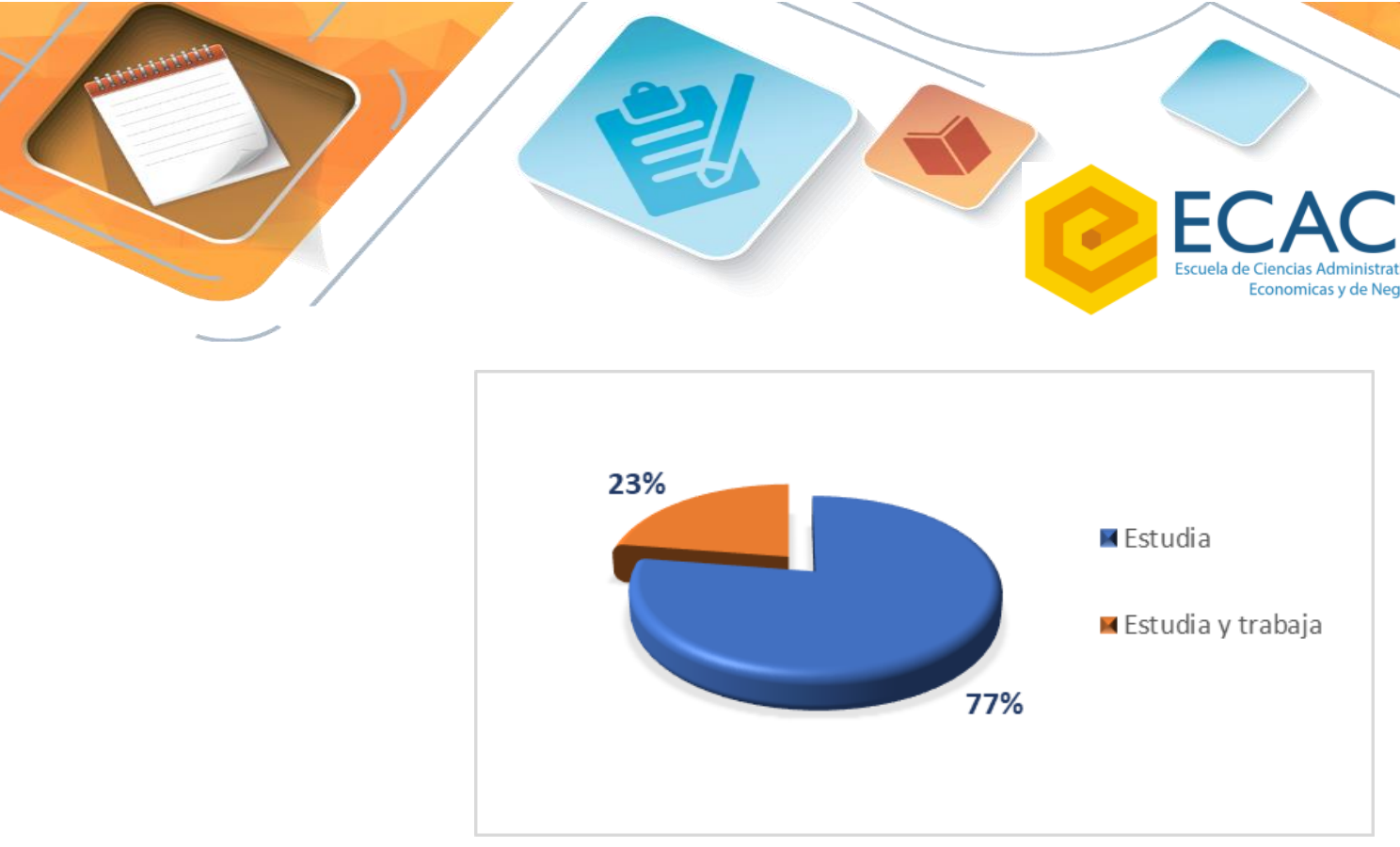

Gráfica 5. Situación actual de los estudiantes encuestados

Otro de los resultados del diagnóstico se relaciona con la situación socioeconómica del hogar del estudiante, convirtiéndose en un factor determinante para la propensión del ejercicio de actividades laborales en los núcleos familiares rurales; cabe mencionar que dicha hipótesis en ocasiones no es válida, puesto que se presentan diferentes motivaciones dentro de los estudiantes para ejercer una determinada actividad independiente del nivel de vida. Sin embargo, en la zona rural de Pasto, el 23\% los estudiantes encuestados además de encontrarse desarrollando sus estudios secundarios, combinan sus actividades académicas con el ejercicio de actividades laborales a fin de obtener un ingreso económico.

Según el Sistema Nacional de indicadores Educativos para los nieles pre escolar básica y media en Colombia (2014), el nivel de escolaridad promedio a nivel nacional en las áreas rurales es bajo; situación que genera desempleo y obliga a que más personas, sobre todo los jóvenes se dediquen a labores del campo mal remuneradas, actividades de construcción y oficios domésticos para las mujeres. 


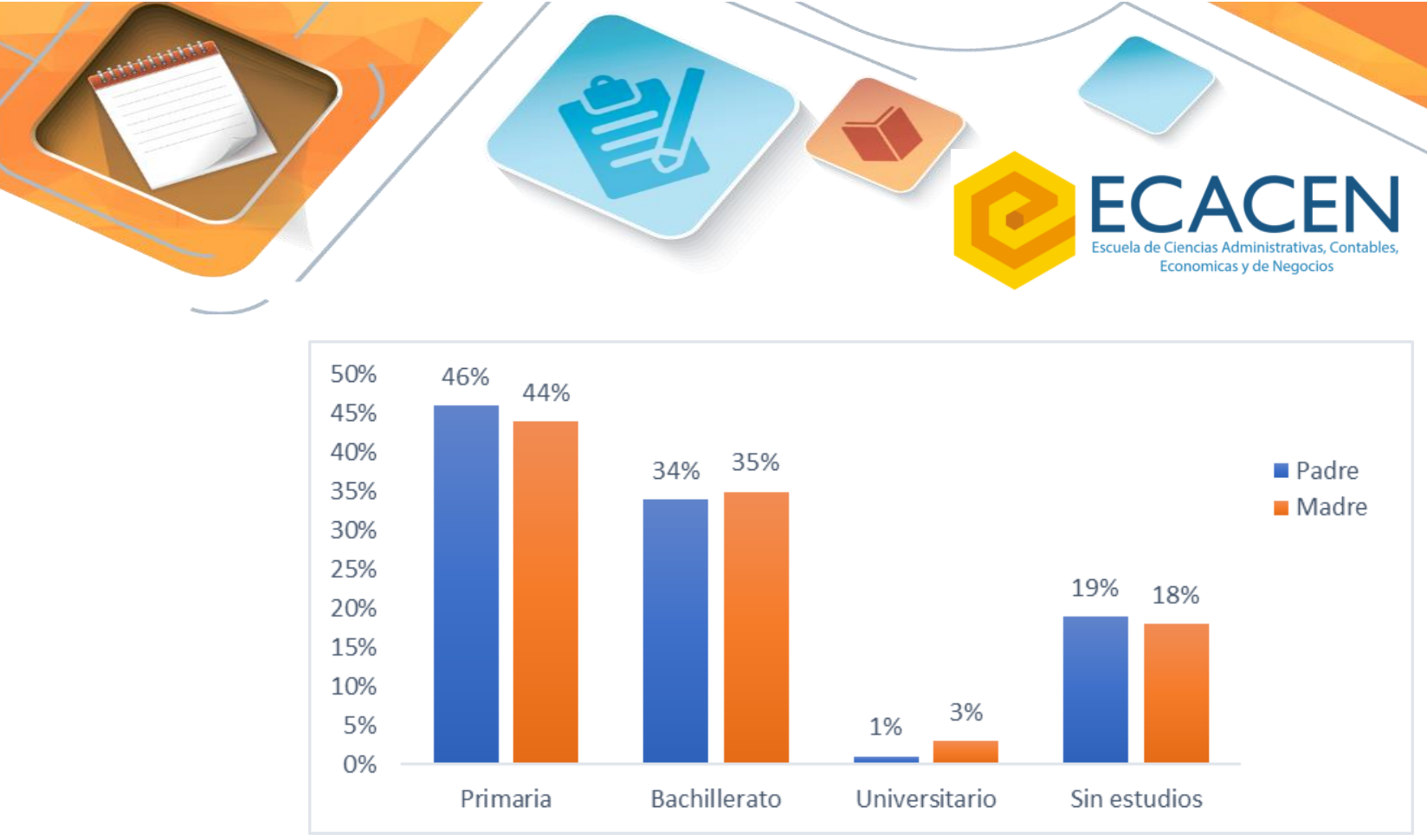

Gráfica 6. Máximo nivel de estudios del padre y madre

En un proceso de formación juega un papel fundamental su núcleo familiar, es por ello que otro de los aspectos indagados tiene que ver con el nivel educativo de los padres de los estudiantes consultados. Los resultados obtenidos con referencia al nivel educativo tanto de los padres como madres de los estudiantes participantes en la investigación reportan que el 46 \% han sido participes de la formación en educación básica primaria, el 34 \% presenta una formación de Básica secundaria y media vocacional y el $1 \%$ del género masculino manifiestan haber sido participes de una formación profesional y es de resaltar que el 3\% de las madres consultadas cuentan con una formación profesional. Un dato relevante para el desarrollo de la investigación es que entre un $18 \%$ al $20 \%$ de las madres y padres de familia no cuentan con estudios argumentando que dicha situación se debe a múltiples factores.

Al respecto, López y Trejos, (2013) comentan que, la formación académica, representa la posibilidad para que la sociedad en general pueda acceder progresivamente a nuevas etapas de desarrollo. Desde lo social, el emprendimiento es muy importante y conlleva acciones representativas, para disminuir la pobreza y convertir a los estudiantes en agentes productivos involucrándolos como potenciales emprendedores en la economía local. 


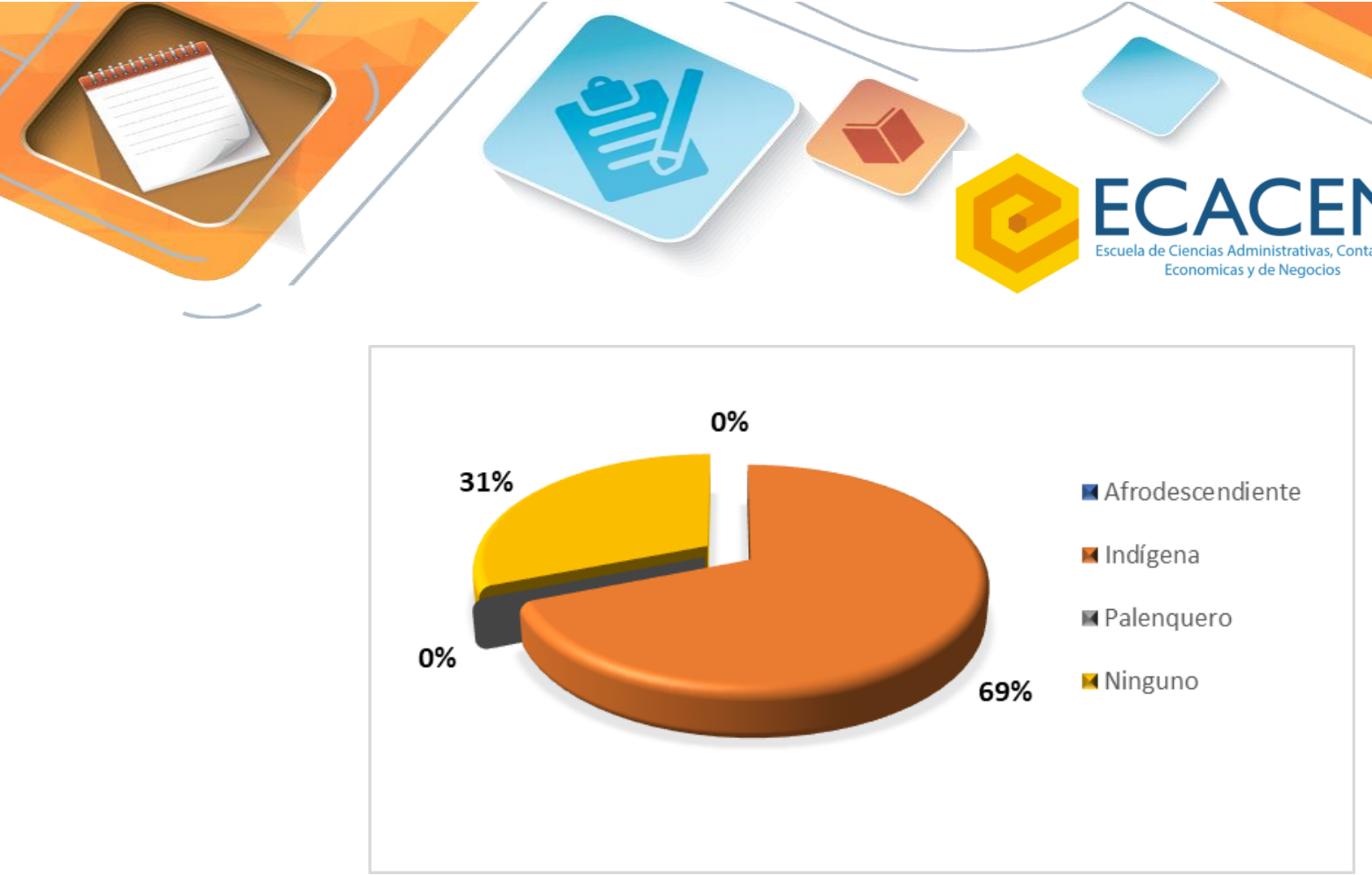

Gráfica 7. Grupo étnico al que pertenece

Una de las características de los sectores rurales colombianos es la existencia de una diversidad cultural y étnica, para el caso específico de la población objeto de estudio se encuentra que el $69 \%$ de los estudiantes pertenecen a un resguardo indígena, y el 31 \% restante no reportan dicha situación. Pese a lo anterior en cada una de las Instituciones se respeta la diversidad como un derecho consagrado dentro de la constitución Nacional y desde esa situación se proyectan las actividades académicas y de emprendimiento. 
